\title{
THE PHOTOCHRONOGRAPH.
}

The photochronograph, and its application to star transits. By J. G. Hagen, S. J., and G. A. Fargis, S. J., Georgetown College Observatory. Georgetown, D. C., 1891. 4to, pp. 36.

THE authors of the above publication are the first to lay before the astronomical world a solution, or at least a partial solution, of the very important problem of meridian transit photography. The instrument they have employed consists essentially of an electromagnetic shutter or "occulting bar," which can be attached to the eye-end of a transit instrument or meridian circle. The apparatus is so arranged that the current from a break-circuit clock moves the occulting bar every second in such a way that the image of a star in transit is impressed for a moment upon a photographic plate mounted behind the bar. A line of "star-dots" can afterwards be developed upon the plate. In order to refer the dots to the collimation axis of the instrument, a glass reticle plate, ruled with one vertical reference line, is permanently fixed in the tube, directly in front of the sensitized surface, and in contact with it. After the star transit is over, it is easy to impress the line upon the sensitized plate, by allowing the light of a lantern to fall for a moment upon the objectglass of the telescope. While this is being done, the line of star dots is shielded from the light by the occulting bar, now permanently interposed between the dots and the light. This method of impressing the reference line upon the plate is excellent, and is further improved by ruling the line with a break in the middle, so that none of the dots can possibly be " occulted" by the line itself. The plates are measured with a micrometric apparatus, by means of which it is easy to determine the instant of the passage of the star across the reference line.

The process thus very briefly outlined is given by the authors with all possible detail ; even the preliminary apparatus, subsequently discarded as imperfect, being carefully described. Other experimenters in the same field should therefore be greatly aided by the present work. In this connection it is proper to refer to the earlier observations of L. M. Rutherfurd, of New York, who successfully employed an arrangement essentially similar to the photochronograph many years ago.* In the collection deposited by Mr. Ruther-

* B. A. Gould, Memoirs of the National Academy of Sciences, vol. iv., p. 175 .

L. M. RUTHERFuRd, American Journal of Science and Arts, vol. iv., Dec., 1872. 
furd at Columbia College* are many negatives showing lines of star-dots, together with micrometric measures of the same.

We shall now enter into a somewhat more careful examination of some of the statements contained in the book, taking them up in order. It is difficult to see why only one vertical line has been used on the reticle plate. The authors refer to their reason for this (p. 12), but without anywhere definitely stating it. One would think the presence of several vertical lines would offer a valuable control of possible irregular expansions of the film during development. Nor would there be any compensating disadvantage, for the admirable device of breaking the lines in the middle would prevent any interference with the star-dots. The effect of irregular distortion of the film would not be eliminated by the method of measurement (p. 24). It is gratifying to find (p. 13) that no trouble was experienced from a jarring of the instrument by the regular beats of the occulting bar.

Probably the most important difficulty of the method is touched upon by the authors in speaking of collimation (p. 17). In fact, it may safely be said that the photographic transit instrument will not be applicable to the finest fundamental work, until it becomes possible to determine the collimation and level constants photographically; without reversal in the Y's, and without the use of the hanging level. In all the observations so far made, the collimation constant has been determined from reversals alone, and the hanging level has always been employed. A very interesting remark occurs (p. 18) in connection with personal equation. By watching the occulting bar through an eye-piece while a star is in transit, the existence and effect of the observer's personal equation become very obvious.

We now come to Part II. of the book, which treats of the reduction of the observations. This part is the work of J. G. Hagen, S.J.; the first part, in which the instrument and methods are described, being by G. A. Fargis, S.J. The screw of the measuring micrometer has been examined for both periodic and progressive errors, according to the usual methods. The author very justly concludes that it is advisable to determine the screw value separately from each plate, though errors due to an oblique mounting of the plate in the tube would not be eliminated thereby, as seems to be implied in the text $(p .22, c)$. The adopted method of measurement, by which the dots are taken in corresponding pairs at nearly equal distances on both sides of the central line, has much in its favor. With regard to the example of a series of transits

* J. K. REes, Annals of the New York Academy of Sciences, vol. vi., June, 1891. 
(p. 34) it may be said that the data are not sufficient to draw conclusions of a very definitive character, beyond the fact that the method gives results of very satisfactory accuracy. The azimuth constants for the evening (two in number) and the collimation constant have been derived from the fifteen observations themselves. Their values are stated to be the "most probable" ones. If they have been obtained by a least-square reduction in which the clock-rate was ignored, it is not remarkable that the final residuals show no evidence of a clock-rate (p. 35).

In conclusion, we may accord to the authors of this book the credit of having invented and made public a photographic method by which meridian transits may be observed with high accuracy, and with a complete freedom from personal equation. If there is a weak point, it will be found in the determination of the instrumental constants. The many other important purposes for which the photochronograph is very well adapted we shall not touch upon in this place. Some of them have already been described in print, and many others will doubtless shortly come into prominence.

Columbia College, New York, 1891, October.

HAROLD JACOBY.

\section{NOMENCLATURE OF MECHANICS.}

BY T. . W. WRIGHT, PH.D.

THE nomenclature of mechanics is in a somewhat confused condition. There is some excuse for this because the science is one of the oldest, and at the same time one of the most progressive, as it certainly is the most comprehensive. New terms are being introduced, others are being suggested to take the place of old ones; but the naturally conserrative cling to the old, and hence we have a duplication, and in some cases a triplication of names for the same thing. At the threshold we are met by a difficulty. How shall we define mechanics? Originally the science of machines, it is by some defined as the science of matter and motion. By others the term dynamics is applied to the science of matter and motion, and the term mechanics is discarded. The tendency at present seems to be in the direction of the latter method. The science is founded on three principles or laws laid down by Newton. These laws were originally enunciated in Latin, and the number of translations is very great. Here is a source 